\title{
Políticas sociales, concentración geográfica y sistemas electorales*
}

\author{
Ignacio Jurado \\ Universidad de Manchester \\ Sandra León \\ Universidad de York
}

\section{Resumen}

La literatura sobre el Estado de Bienestar ha asumido frecuentemente que los beneficiarios de políticas sociales se distribuyen homogéneamente, ignorando que el gasto público pueden tener receptores concentrados en determinadas regiones. En ese caso, las políticas sociales nacionales son en realidad políticas con fuertes sesgos regionales. Ante esto, un político racional tendrá en cuenta la distribución geográfica de los destinatarios antes de formular una política. En este artículo aplicamos este marco teórico para analizar los niveles de provisión de dos políticas que tienden a ser consideradas como políticas nacionales: las prestaciones por desempleo y las pensiones de jubilación. Para ello, analizamos cómo afecta la concentración geográfica de los beneficiarios, teniendo en cuenta los distintos sistemas electorales. Los resultados muestran que cuando los destinatarios están concentrados geográficamente, los sistemas electorales mayoritarios incentivan su provisión, mientras que esta decrece en sistemas electorales proporcionales.

Palabras clave: políticas sociales, concentración geográfica, sistemas electorales.

Clasificación JEL: D72, H55.

\section{Abstract}

The literature on the welfare state has often assumed that social policy recipients are homogenously distributed across a country. It has been overlooked that public expenditures may have potential beneficiaries that might be concentrated in certain regions. Therefore, national social policies, which are not geographically targeted, are actually policies with strong regional biases. Given this, rational politicians should take into account the geographic distribution of recipients before formulating a policy. We apply this theoretical framework to analyze the provision of unemployment and old age benefits, which tend to be considered as paradigmatic national policies. We show that the recipients' geographical concentration accounts for different patterns of social policy provision between electoral systems. The empirical analyses show that, when recipients are concentrated, majoritarian electoral systems encourage the provision of these policies, while they decrease in proportional electoral systems.

Keywords: social policies, geographic concentration, electoral systems.

JEL classification: D72, H55.

\section{Introducción}

La literatura de Economía Política ha llegado frecuentemente a conclusiones sobre los niveles de gasto público y su variación a lo largo del tiempo asumiendo que la población es geográficamente homogénea. Así, se ha ignorado que casi todas las 
políticas públicas tienen beneficiarios que no se distribuyen por igual en un país. En concreto, cuando los países industrializados alcanzan un nivel alto de desarrollo, las divergencias regionales suelen aumentar (Ezcurra y Rapun, 2006).

¿Cómo reaccionan los gobiernos a la geografía de los grupos sociales? Esta es la pregunta de investigación central de este artículo. Nuestro análisis muestra que la provisión de políticas nacionales de gasto está determinada por la distribución regional de sus receptores. Este efecto, además, está condicionado por el sistema electoral. Nuestros resultados permiten cuestionar una literatura bien establecida sobre sistemas electorales, la cual sostiene que los gobiernos en sistemas proporcionales proveen mayores niveles de gasto social bajo sistemas electorales proporcionales. Nuestro argumento, por el contrario, es que cuando los destinatarios de las políticas sociales se concentran geográficamente, los incentivos para proporcionar estas políticas son más fuertes en los sistemas electorales mayoritarios. Este artículo pone a prueba esta hipótesis con datos de 15 países de la OCDE para el periodo 1983-2004.

El artículo tiene la siguiente estructura. La sección segunda revisa los argumentos sobre sistemas electorales y gasto social e introduce la hipótesis principal. En la sección tercera, se presentan los datos y el método, mientras que el apartado cuarto muestra los resultados. Por último, en la sección quinta desarrollamos algunas conclusiones y la agenda de investigación que se deriva de nuestros resultados.

\section{Políticas sociales, sistemas electorales y concentración geográfica}

¿Cuándo proveen los gobiernos niveles más altos de políticas sociales? La literatura académica ha tratado de arrojar luz sobre esta cuestión durante mucho tiempo. Trabajos clásicos en Ciencia Política enfatizan el rol de las divisiones entre clases sociales para explicar los niveles de redistribución (Lipset y Rokkan, 1967). Además, la literatura ha proporcionado explicaciones relacionadas con la estructura corporativista del Estado (Grant, 1985; Schmitter, 1974), la apertura comercial de las economías nacionales (Katzenstein, 1985), la fuerza del movimiento obrero y los sindicatos (Esping-Andersen, 1990; Korpi, 1978; Korpi, 1983), o la estructura industrial del país (Hall y Soskice, 2001).

En los últimos años, la explicación que ha recibido mayor atención en la disciplina se centra en el sistema electoral. Esta literatura argumenta que mientras los sistemas electorales proporcionales dan incentivos para la provisión de políticas sociales, en los sistemas electorales mayoritarios la distribución se hace fundamentalmente mediante políticas distributivas, compuestas por transferencias específicas, bienes públicos locales y pork barrel, entre otros (Persson y Tabellini, 2000, 2003; Lizzeri y Persico, 2001; Milesi-Ferretti et al., 2002; Grossman y Helpman, 2005).

Varios argumentos teóricos justifican esta perspectiva. En primer lugar, esta literatura da mucha relevancia a si la competencia electoral tiene lugar en uno solo o en múltiples distritos. La división en distritos cambia completamente la naturaleza de las elecciones. Simplificando hasta el extremo, en un sistema proporcional de un 
único distrito los partidos han de maximizar su porcentaje de votos en el conjunto del país. Cuanto mayor sea el número de votos que obtengan, más representación tendrán. Por el contrario, en los sistemas mayoritarios los partidos maximizan su representación ganando en el mayor número de distritos. Esto lleva a que en los sistemas proporcionales los partidos compiten por votos en todo el país, mientras que en los mayoritarios se centran en distritos específicos (Persson y Tabellini, 2000, 2003; Chang et al., 2008). Así, en términos de las políticas de gasto, en un sistema de representación proporcional los partidos basarán sus estrategias distributivas en políticas nacionales y sociales universalistas que no discriminan geográficamente. En cambio, en un sistema mayoritario, los partidos dirigirán el gasto a aquellos distritos que son decisivos para el resultado final de las elecciones. Los partidos asignarán recursos exclusivamente en ellos, ignorando los distritos donde va a ganar o perder con seguridad. Para ello, utilizarán pork barrel y transferencias directas al distrito.

La forma en que se desarrolla la competición electoral es también diferente entre sistemas electorales. En los sistemas proporcionales, los electores siempre votan por una lista. En cambio, en los sistemas electorales mayoritarios donde hay múltiples distritos, los votantes habitualmente votan por un candidato. Milesi-Ferretti et al. (2002) sostienen que en los sistemas proporcionales los partidos terminan representando a grupos socioeconómicos, mientras que en los sistemas mayoritarios, los representantes de un distrito tienen incentivos a formar coaliciones de apoyo más heterogéneas socialmente en sus circunscripciones. Además, cuando el sistema electoral se basa en listas, los representantes tienen incentivos a apoyar políticas nacionales que maximizan el voto a la lista de su partido, y no tienen incentivos a desviarse. En cambio, cuando el voto es al candidato, los representantes tendrán mayor interés en cultivar el voto personalista dirigiendo políticas que beneficien exclusivamente a sus distritos.

Estos argumentos indicarían que el gasto social y universalista sería mayor en sistemas proporcionales. A pesar de estas predicciones, la evidencia empírica sobre la relación entre sistema electoral y composición del gasto público no es concluyente (Rickard, 2009; Franzese y Nooruddin, 2004). Desde nuestro punto de vista, esto se debe a que la literatura ha ignorado los factores geográficos asumiendo implícitamente dos cosas. En primer lugar, se ha dado por sentado que la demanda por una determinada política social es siempre homogénea en todo el país. Esto implica que si se comparan dos países, uno donde la política social tiene receptores dispersos, y otro donde los receptores se concentran geográficamente, la demanda será similar. Sin embargo, nuestro argumento es que estas situaciones son muy diferentes, ya que la distribución geográfica de los beneficiarios da forma a la intensidad y la relevancia de las demandas de los grupos sociales.

En segundo lugar, y de modo más relevante, todos estos autores trazan una línea divisoria entre las políticas sociales y las distributivas, siendo las primeras universalistas y nacionales, mientras que las segundas están conformadas por trasferencias con un sesgo local. Esta dicotomía implica que cada política sirve para objetivos electorales muy diferentes (Franzese y Nooruddin, 2004; Persson y Tabellini, 2000). El pork barrel se asocia con motivaciones geográficas concretas, mientras que las 
políticas sociales y de bienestar se asocian con un enfoque nacional amplio. Así, cuando los gobernantes están interesados en votantes con una ubicación específica, utilizarán pork barrel, políticas de clientelismo, bienes públicos locales y otras políticas distributivas. Por el contrario, cuando están interesados en tener un impacto electoral en el conjunto del país utilizarán las políticas sociales.

Sin embargo, aunque este marco general asume que las políticas distributivas son la única herramienta de gasto disponible cuando hay incentivos geográficos, la realidad es que el impacto de las políticas de bienestar puede ser muy territorializado. La dependencia de las políticas sociales varía mucho a lo largo de un país. Por lo tanto, el argumento de que estas políticas son nacionales no se sostiene si ciertas regiones las demandan más que el resto del país.

De hecho, este es un patrón creciente en muchos países. Una vez que se alcanza un cierto nivel de desarrollo, la divergencia económica regional tiende a aumentar (Ezcurra y Rapun, 2006). Por ejemplo, el PIB per cápita en el sureste de Inglaterra es casi el doble que en los condados industriales del norte. Bélgica tiene una fuerte división entre Valonia y Flandes. Aún más evidente es la fractura económica en Italia entre el Norte y el Sur, o en Alemania entre Oeste y Este. Incluso en países con grandes estados de bienestar como Finlandia, nos encontramos con niveles de variación regional importantes que implican que los beneficios de las políticas sociales se reparten de modo desigual entre las diferentes regiones del país.

Estos patrones regionales emergen con más fuerza si analizamos beneficiarios de políticas específicas. En particular, los dos que se estudian en este artículo: las prestaciones por desempleo y las pensiones de jubilación. Si nos fijamos en la distribución de estos receptores, con frecuencia vemos que están lejos de ser un grupo «nacional». Los países industrializados han experimentado un proceso de territorialización de estos grupos. En Italia, la mayor parte del desempleo se encuentra en la mitad sur del país. Después de la reunificación de Alemania, el desempleo se considera principalmente un fenómeno del Este. España, Bélgica y Turquía, por poner otros tres ejemplos muy diferentes, también muestran fuertes patrones regionales de desempleo. Como decíamos anteriormente, esta es una tendencia creciente. Overman y Puga (2002) muestran que las regiones que tenían una baja tasa de desempleo con respecto a la media europea en 1986 tendieron a mantener o reducir su tasa de desempleo en la década siguiente, mientras que las regiones que tenían una alta tasa de desempleo en 1986 tienden a tener una mayor tasa de desempleo en 1996.

Este fenómeno de regionalización, aunque muy relevante en relación con el desempleo, se extiende también a los pensionistas. Los procesos de urbanización y el papel predominante de las zonas urbanas en la actividad económica han dado lugar a un patrón de concentración de las personas de mayor edad en las zonas rurales. La Regional Statistics de la OCDE muestran, por ejemplo, que en Francia, Italia o Portugal las zonas rurales tienen dos o incluso tres veces más población por encima de 65 años que las zonas urbanas. Diferencias significativas también se pueden encontrar en Finlandia, Australia o Estados Unidos. Igualmente, la po- 
blación en edad escolar se distribuye muy asimétricamente. En el sur de España el porcentaje de población menor de 14 años es un 50 por 100 más alto que en las regiones del norte.

En resumen, los beneficiarios de las políticas sociales pueden estar muy concentrados. En consecuencia, políticas que se suelen considerar como nacionales y universalistas tendrán en realidad fuertes sesgos regionales. Este fenómeno pondría en cuestión algunas de los supuestos de la literatura que relaciona sistemas electorales y políticas sociales. En particular, la predicción de que los políticos en sistemas proporcionales formulan mayores niveles de políticas sociales para competir electoralmente en el conjunto del país, mientras que en los sistemas mayoritarios utilizan otras políticas para competir en distritos específicos.

El argumento que testamos en este artículo es si, cuando los receptores están concentrados geográficamente, los incentivos para proporcionar una política son mayores en sistemas electorales mayoritarios que en proporcionales. En los sistemas mayoritarios, los partidos pueden renunciar a algunos votos en los distritos que ganan con seguridad para tratar de vencer en aquellos más competitivos. Si un grupo social se concentra en estos distritos, los partidos buscarán proporcionar aquellas políticas sociales que benefician relativamente más a estas circunscripciones. En ese caso, no será necesario que recurran a bienes públicos locales u otras transferencias distributivas. Gran parte del gasto en esa política social se canaliza implícitamente a ese distrito, sencillamente porque el grupo social beneficiario se concentra en él. Esto debería aumentar los incentivos para proveer la política social, en comparación con un sistema proporcional, donde la distribución geográfica de los beneficiarios debería ser irrelevante.

Por tanto, no cuestionamos la lógica que subyace en la literatura de sistemas electorales. En su lugar, nos cuestionamos la confusión entre objetivos electorales y las políticas de gasto que los cumplen. Milesi-Ferretti et al. (2002) y Persson y Tabellini (2003) asumen que si los partidos quieren localizar gasto público a costa del resto de distritos, la única forma es a través de políticas distributivas dirigidas al distrito. Sin embargo, cuando un distrito es particularmente sensible a una política social (ya que en él se concentran un gran número de receptores), los representantes políticos recibirán grandes beneficios electorales en ese distrito si proveen esa política social. En conclusión, cuando los receptores de una política social están concentrados, esperamos que las predicciones de la literatura de sistemas electorales se reviertan. Los sistemas proporcionales, caracterizados como países donde los partidos compiten en un único distrito nacional, darán menos incentivos que los sistemas mayoritarios para formular políticas sociales. En estos últimos, los partidos en el gobierno se apoyarán en políticas sociales como instrumentos distributivos con un impacto geográfico concreto. 


\section{Datos y métodos}

\subsection{Variables dependientes}

Como se ha explicado anteriormente, nuestro argumento es que, bajo determinadas circunstancias geográficas, las políticas sociales tienen un sesgo regional que las hacen más atractivas en sistemas electorales mayoritarios. Para testar esta hipótesis, analizamos dos políticas nacionales que con frecuencia tienen beneficiarios regionalizados: las prestaciones por desempleo y las pensiones de jubilación ${ }^{1}$. Ambas variables se miden como porcentaje del PIB y han sido extraídas de la OECD Social Expenditure Database (2007).

\subsection{Variables independientes principales}

Para explorar la relación entre las políticas sociales y la concentración de receptores, necesitamos una medida de su dispersión geográfica. Hemos calculado dos índices diferentes.

La primera medida es un Índice de Herfindahl Transformado de concentración de grupos sociales (IHT). El índice se calcula ponderando el desempleo regional (o la tasa de personas mayores de 65) por el tamaño de la región (medida como población activa total en el primer caso y población total regional para el segundo). Así, el índice se obtiene de:

$$
I H T=\frac{1}{1-V} \sum_{i=1}^{N} x_{i}\left(d_{i}-\mu_{i}\right)^{2}
$$

$$
\text { donde (2) } V=\frac{1}{1-\sum_{1=1}^{n} x_{i}^{2}}
$$

siendo $d_{i}$ el porcentaje regional del grupo social sobre la población regional, $\mu_{i}$ el porcentaje nacional del mismo grupo sobre el conjunto de la población, y $x_{i}$ el tamaño de cada región $i$.

La segunda medida se obtiene de Ellison y Glaeser (1997). Estos autores proporcionan un índice de concentración geográfica industrial, que se puede utilizar también para la concentración de los grupos sociales. El índice resultante (EG) es el siguiente:

$$
\text { (3) } E G=\frac{\sum_{i}^{N}\left(s_{i}-x_{i}\right)^{2}-\left(1-\sum_{i=1}^{n} x_{i}^{2}\right) \frac{1}{Z}}{\left(1-\sum_{i=1}^{n} x_{i}^{2}\right)\left(1-\frac{1}{Z}\right)}
$$

${ }^{1}$ Para el análisis de las prestaciones por desempleo, también realizamos los modelos utilizando las tasas de sustitución de SCRUGGS (2005). Los resultados son robustos y están disponibles bajo petición. 
donde $s_{i}$ es la el porcentaje del conjunto del grupo social que vive en la región $i$, $x_{i}$ es la proporción de la población nacional que vive en la región $i, \mathrm{y} z$ es el porcentaje del grupo social sobre la población nacional.

Utilizamos ambos índices para calcular la concentración del desempleo y de gente mayor de 65 años. Para los índices de desempleo, utilizamos las tasas regionales y nacionales de desempleo. Para las medidas de concentración de pensionistas, utilizamos el porcentaje regional y nacional de las personas mayores de 65 años. Los datos se han tomado de las Eurostat Regional Statistics (para los países europeos), y de las estadísticas regionales de la OCDE (para los países no europeos) ${ }^{2}$. Ambas medidas, IHT y EG, son comparables entre países. Con algunas excepciones, producen clasificaciones similares. La correlación entre índices es alta, 0,77 en el caso de los índices de desempleo, y 0,68 en el caso de la concentración de mayores de 65.

Según nuestra hipótesis, esperamos que el efecto de ambos índices sobre las políticas sociales esté supeditado a la naturaleza del sistema electoral. Para comprobarlo, necesitamos interactuar estos índices con una medida de proporcionalidad del sistema. Consistentemente con la literatura, utilizamos el logaritmo de la magnitud media de distrito (ln magnitud de distrito) ${ }^{3}$. Numerosos trabajos han mostrado que la principal característica que define la proporcionalidad del sistema electoral no es la regla electoral, sino la magnitud media de los distritos (Taagepera y Shugart, 1989; Lijphart, 1990). Cuando el número de escaños distribuidos en un distrito es bajo, la norma electoral apenas importa, pues la asignación siempre tiene un fuerte sesgo mayoritario. Así, los países más mayoritarios, como el Reino Unido o los Estados Unidos, con un sistema de distrito uninominal tienen valor cero en esta medida. En un país como Irlanda, donde hay un sistema de voto transferible (STV) en distritos de cuatro miembros, la medida ln magnitud de distrito tiene un valor de 1,38. España, un país con un sistema en teoría proporcional, pero donde existen muchos distritos pequeños que introducen un fuerte sesgo mayoritario, tiene un valor de 1,9 en esta medida. En el otro extremo, los Países Bajos, con un sistema perfectamente proporcional en un distrito único nacional de 150 miembros, tienen un valor de 5,01 .

${ }^{2}$ Las OECD Regional Statistics considera como regiones las NUTS 2 de Eurostat en los países europeos, los estados en EE.UU, las provincias en Canadá, las regiones en Japón y los Estados y Territorios en Australia.

${ }^{3}$ La magnitud promedio del distrito es obtenida de la base de datos de sistemas electorales de GOLDER (2007). 


\subsection{Método}

Para testar la hipótesis, utilizamos datos de 15 países de la $\mathrm{OCDE}^{4}$. Nuestro panel cubre, en el caso de España y Bélgica, el período 1983-2004. En el caso de Portugal el panel cubre tan sólo ocho años (1997-2004). En promedio, cada modelo utiliza alrededor de 13 observaciones por país.

Nuestras estimaciones incluyen panel corrected standard errors, efectos fijos y una variable dependiente retardada (Beck y Katz, 1994). Como incluimos varias variables institucionales, cuyo efecto podría ser absorbido por los efectos fijos, hemos realizado también los análisis utilizando el método de fixed effects vector decomposition, propuesto por Plümper y Troeger (2007). Este método permite separar los efectos institucionales de los efectos fijos. Los resultados son muy robustos y los hallazgos principales permanecen invariables ${ }^{5}$.

\subsection{Variables de control}

\section{Variables económicas}

Incluimos dos variables de capacidad financiera del gobierno. En primer lugar, el nivel de deuda pública del año anterior controla por las restricciones a las que un gobierno se enfrenta al tomar sus decisiones presupuestarias. Altig y Davis (1989) muestran cómo los niveles de deuda afectan a los cálculos distributivos de los gobiernos. Por otro lado, la tasa de interés es un indicador del precio de aumentar el gasto público sin aumentar los ingresos.

También controlamos por el PIB, tanto en su tasa de crecimiento con en su valor total. El incremento del PIB es un control económico muy estándar para dar cuenta de los efectos del crecimiento sobre las decisiones políticas de los gobiernos. El PIB per cápita (medido logarítmicamente) controla por el efecto de la ley de Wagner. Si esta es cierta, esperaríamos que los países más ricos redistribuyan más.

Por último, incluimos en nuestros modelos la apertura comercial, medida como el comercio total (suma de importaciones y exportaciones) sobre el PIB. Existen dos posibles efectos de la apertura comercial en las políticas sociales. Algunos autores predicen que niveles altos de comercio se asocian con mayores niveles de gasto social. Éste actuaría como colchón ante las volatilidades de los mercados internacionales (Katzenstein, 1985; Rodrik, 1998). Por otro lado, otros autores predicen que la globalización obliga a los países a disminuir sus niveles de gasto social para competir mejor en los mercados internacionales (Tanzi, 1997).

${ }^{4}$ Los países de nuestra muestra son: Alemania, Australia, Austria, Bélgica, Canadá, España, Estados Unidos, Finlandia, Francia, Grecia, Italia, Japón, Países Bajos, Portugal, Reino Unido y Suecia.

${ }^{5}$ Estos resultados están disponibles bajo petición a los autores. 


\section{Variables demográficas}

Incluimos en nuestros modelos controles por niveles de desempleo (como porcentaje de la población activa) y la proporción de población mayor de 65 años (medida como porcentaje de la población total) ${ }^{6}$. Además, incluimos los términos cuadráticos de ambas variables para comprobar si existen o no efectos no lineales.

\section{Variables políticas}

Los modelos incluyen una variable de gobierno de izquierda, medida como el porcentaje de ministros que pertenecen a partidos de izquierda ${ }^{7}$. La expectativa es que los gobiernos de izquierda redistribuyan más. Muchos trabajos han mostrado que las variables a nivel de partido son relevantes para explicar las decisiones de gasto en los países (véase, por ejemplo, Boix, 1998; Huber y Stephens, 2001; Bradley et al., 2003; Rueda, 2007).

La participación electoral en las elecciones anteriores también se incorpora en los análisis. Dado que los ciudadanos de bajos ingresos son menos propensos a votar, los partidos deberían tener más incentivos para proporcionar políticas redistributivas cuando la participación es más alta (Kenworthy y Pontusson, 2005; Nelson, 1999). Por otro lado, existe una amplia literatura sobre los ciclos electorales que dice que los partidos tienen más incentivos para incrementar las políticas sociales y redistributivas justo antes de las elecciones (Alesina y Roubini, 1992; Alesina et al., 1998; Hibbs, 1977). Por ello, incluimos una variable dummy para el año electoral. Para ambas variables, utilizamos las elecciones a la cámara baja (a excepción de los Estados Unidos, donde se utilizan las elecciones presidenciales).

Finalmente, numerosos trabajos han recalcado el efecto de la fragmentación de los gobiernos en el gasto (Jones et al., 1997). Por eso, incluimos dos dummies: una para gobiernos de coalición y otra para gobiernos en minoría.

\section{Variables institucionales}

Incluimos dos tipos de controles institucionales. En primer lugar, los modelos incorporan una medida de coordinación de la negociación salarial (Visser 2009) ${ }^{8}$. La predic-

${ }^{6}$ El supuesto subyacente es que todos los desempleados reciben prestaciones por desempleo y todos los ancianos reciben pensiones de jubilación. Aunque ambos grupos pueden contener individuos que no reciben tales prestaciones, se trata de una representación bastante precisa del número de personas cubiertas por la política social.

${ }^{7}$ Esta medida está ponderada por el número de días del año en que el miembro del gabinete ocupa el cargo. Se obtiene de ARMINGEON (2009).

${ }^{8}$ La medida se basa en la de KENWORHTY (2001) y mide de 1 (negociación fragmentada a nivel de empresa) a 5 (acuerdos vinculantes a nivel nacional entre patronal y sindicatos). 
ción es que las prestaciones por desempleo y jubilación aumentan cuando hay instituciones corporativas. El segundo control institucional es un índice de restricciones institucionales elaborado por Schmidt (1996). Este índice mide el grado en que hay instituciones y actores con poder de veto que pueden bloquear iniciativas de los gobiernos.

\section{Resultados}

Presentamos dos conjuntos de modelos. En el primer conjunto (Cuadro 1), testamos la hipótesis utilizando las prestaciones por desempleo como variable dependiente. El Cuadro 2 replica los modelos con las pensiones de jubilación como variable dependiente. En cada serie de modelos utilizamos los dos índices de concentración.

Antes de entrar en las conclusiones principales, es necesario algún comentario sobre las variables de control. Un primer resultado sorprendente es el diferente efecto de las variables demográficas. En los modelos sobre prestaciones de desempleo, el nivel de los receptores de la política (la tasa de desempleo) muestra un efecto en forma de U inversa. Por el contrario, la tasa de personas de edad avanzada (mayores de 65) tiene un efecto en forma de $\mathrm{U}$ en la provisión de pensiones de jubilación. Entre las variables económicas, la tasa de interés muestra el signo esperado, y, en algunos casos, con niveles convencionales de significatividad. Los niveles de PIB per cápita se relaciona negativamente con la provisión de políticas sociales, lo que supone evidencia contraria a la ley Wagner. El crecimiento del PIB tiene un efecto significativo en la menor necesidad de políticas sociales. Por otra parte, nuestros resultados son ambiguos respecto a los efectos de la globalización. La variable de apertura comercial suele tener signo negativo y significativo, salvo en el modelo 1.1 en que el efecto es positivo.

Algunas variables tienen diferentes efectos en los análisis sobre prestaciones por desempleo y en los de pensiones. La deuda del año anterior, por ejemplo, muestra un signo negativo en la regresión de prestaciones por desempleo. Sin embargo, y sorprendentemente, tiene un impacto consistentemente positivo y significativo sobre las pensiones. Esto significa que mientras las restricciones financieras son un obstáculo para las prestaciones a grupos de ingresos bajos, como los desempleados, no parecen restringir el nivel de beneficios a un grupo de mayor ingreso como los pensionistas.

Entre las variables políticas, no encontramos efectos partidistas sobre las transferencias sociales. Esto apoyaría investigaciones recientes que afirman que sólo bajo ciertas condiciones los partidos de izquierdas redistribuyen más (Rueda y Pontusson 2000). Asimismo, no encontramos evidencia de ciclos electorales, siendo la variable año electoral no significativa. Por el contrario, sí encontramos evidencia de que una mayor participación electoral disminuye la provisión de pensiones, aunque, contrariamente a nuestras expectativas, no hay un efecto sobre las prestaciones por desempleo. En este sentido, Pontusson y Rueda (2010) proporcionan evidencia empírica de que cuando la participación electoral es alta, sólo los partidos de izquierda tienen más incentivos para aumentar las políticas redistributivas. Esto podría explicar por qué no encontramos un efecto positivo. En algunos análisis suplementarios (no mostrados), el efecto interactivo es significativo. 
Por último, con respecto a las variables institucionales, los resultados no son siempre significativos, probablemente debido a la inclusión de efectos fijos. En cualquier caso, cuando alcanzan la significatividad, las restricciones institucionales reducen la capacidad de los gobiernos para aumentar los beneficios sociales. Igualmente, la coordinación salarial tiene un signo negativo. Esto apoya la perspectiva de que el corporativismo solo protege a los insiders (Rueda 2007)9.

Como se ha dicho anteriormente, la hipótesis principal es testada mediante la interacción entre concentración geográfica de los grupos sociales y el sistema electoral. El Cuadro 1 muestra el efecto en la provisión de prestaciones por desempleo. La magnitud del distrito tiene un efecto positivo y significativo sobre las políticas de desempleo. Esto, en principio, daría apoyo a la literatura convencional sobre sistemas electorales. Los países con sistemas proporcionales proveerían mayores niveles de prestaciones por desempleo. Sin embargo, este efecto está condicionado a que la concentración geográfica de los beneficiarios sea cero. La interacción entre magnitud de distrito y los dos índices de concentración del desempleo es siempre negativa y significativa al 99 por 100 . Esto implica que existen diferencias relevantes entre sistemas electorales en cuanto al efecto de la distribución geográfica de los desempleados. A medida que su concentración aumenta, los sistemas proporcionales proporcionarán niveles más bajos de prestaciones por desempleo. En cambio, en los sistemas electorales mayoritarios los niveles de beneficios por desempleo aumentan cuando los desempleados están concentrados en ciertas regiones.

El Cuadro 2 muestra los resultados de los modelos con la interacción entre la concentración de mayores de 65 y la magnitud de distrito. Una vez más, los resultados confirman claramente la hipótesis de este trabajo. La interacción con el Índice de Herfindahl Transformado (IHT) es un poco más significativa que la que utiliza el índice de Ellison y Glaeser (EG), pero en cualquier caso ambas están muy por encima de los niveles convencionales de significatividad.

En conjunto, estos resultados implican que el efecto positivo de la concentración de receptores es más fuerte en los sistemas electorales mayoritarios que en los proporcionales. Como se ha dicho, esto revierte las predicciones convencionales en la literatura. La expectativa habitual es que la provisión de políticas sociales será mayor en sistemas electorales proporcionales. Sin embargo, esto sólo será así siempre y cuando los beneficiarios no están concentrados geográficamente. Cuando se aglomeran en ciertas regiones, los incentivos para proporcionar políticas sociales son más fuertes en los sistemas electorales mayoritarios. El partido gobernante utilizará las políticas sociales como una política implícita de pork barrel a favor de los distritos donde los destinatarios se concentran.

${ }^{9}$ RUEDA (2007) argumenta que el conjunto de instituciones corporativistas están diseñadas para proteger los intereses de los insiders, los cuales no están interesados en las políticas de desempleo. En su lugar, su preferencia es por políticas de protección del empleo. Por tanto, las instituciones que fortalecen la posición de los insiders conducirán a menos prestaciones por desempleo y mayor protección del empleo. 


\section{CUADRO 1}

ESTIMACIONES DE BENEFICIOS POR DESEMPLEO

\begin{tabular}{|c|c|c|}
\hline Variable Dependiente: Beneficios por Desempleo (\% PIB) & Modelo 1.1 & Modelo 1.2 \\
\hline Variable Dependiente Retardada & $\begin{array}{c}\mathbf{0 , 6 7 7 7 6} * * * \\
0,02892 \\
\end{array}$ & $\begin{array}{c}\mathbf{0 , 6 2 7 9 2} * * * \\
0,03315\end{array}$ \\
\hline Tasa Desempleo & $\begin{array}{c}\mathbf{0 , 0 0 3 8 4} * * * \\
0,00071 \\
\end{array}$ & $\begin{array}{c}\mathbf{0 , 0 0 2 0 8} * * * \\
0,001 \\
\end{array}$ \\
\hline Tasa Desempleo Cuadrática & $\begin{array}{c}-\mathbf{0 , 0 0 7 1 9} * * * \\
0,01048 \\
\end{array}$ & $\begin{array}{c}-\mathbf{0 , 0 1 0 5 1} * * * \\
0,01153 \\
\end{array}$ \\
\hline Mayores 65 Años & $\begin{array}{c}\mathbf{0 , 1 1 9 6} \\
0,239 \\
\end{array}$ & $\begin{array}{c}\mathbf{- 0 , 0 4 6 9 4} \\
0,10674 \\
\end{array}$ \\
\hline Mayores 65 Años Cuadrático & $\begin{array}{c}\mathbf{0 , 0 0 3 7 5} \\
0,541 \\
\end{array}$ & $\begin{array}{l}\mathbf{0 , 0 0 0 2 9} \\
0,00311 \\
\end{array}$ \\
\hline Tasa Interés & $\begin{array}{c}-\mathbf{- 0 , 0 0 7 1 8} \\
0,0013 \\
\end{array}$ & $\begin{array}{l}\mathbf{0 , 0 0 6 4} \\
0,00113 \\
\end{array}$ \\
\hline Deuda Pública (año anterior) & $\begin{array}{c}-\mathbf{0 , 0 1 5 7 2} * * * \\
0,00912 \\
\end{array}$ & $\begin{array}{c}-\mathbf{0 , 0 0 7 8 1} * * * \\
0,00834 \\
\end{array}$ \\
\hline Incremento PIB per capita & $\begin{array}{c}-\mathbf{0 , 2 3 8 3 6} * * \\
0,57377 \\
\end{array}$ & $\begin{array}{c}\mathbf{- 1 , 0 7 7 8 8} \\
0,67292 \\
\end{array}$ \\
\hline Ln PIB per capita & $\begin{array}{c}-\mathbf{0 , 0 0 9 0 2} * * \\
0,00171\end{array}$ & $\begin{array}{c}-\mathbf{0 , 0 1 2 3 7} * * * \\
0,00144\end{array}$ \\
\hline Apertura Comercial & $\begin{array}{c}\mathbf{0 , 0 0 1 0 7} * * * \\
0,00217\end{array}$ & $\begin{array}{c}-\mathbf{0 , 0 0 0 6 3} * * * \\
0,00207\end{array}$ \\
\hline Participación Electoral & $\begin{array}{l}\mathbf{0 , 0 1 7 9 4} \\
0,01159\end{array}$ & $\begin{array}{l}\mathbf{0 , 0 1 4 4 2} \\
0,01326 \\
\end{array}$ \\
\hline Año Electoral & $\begin{array}{c}-\mathbf{0 , 2 0 1 8 2} * \\
0,03938\end{array}$ & $\begin{array}{c}-\mathbf{0 , 1 8 7 7 8} \\
0,04514 \\
\end{array}$ \\
\hline Gobierno Coalición & $\begin{array}{c}-\mathbf{0}, \mathbf{1 7 3 3 7} * * * \\
0,04379 \\
\end{array}$ & $\begin{array}{c}-\mathbf{0 , 1 9 0 1 3} * * * \\
0,054 \\
\end{array}$ \\
\hline Gobierno Minoría & $\begin{array}{c}\mathbf{0 , 0 0 0 7 3} * * * \\
0,0003 \\
\end{array}$ & $\begin{array}{c}\mathbf{0 , 0 0 0 6 2} * * * \\
0,0003 \\
\end{array}$ \\
\hline Gobierno de Izquierdas & $\begin{array}{c}\mathbf{- 0 , 0 9 8 4 7} \\
0,02397 \\
\end{array}$ & $\begin{array}{c}-\mathbf{0 , 1 0 9 5 2} \\
0,0274\end{array}$ \\
\hline Coordinación Salarial & $\begin{array}{c}-\mathbf{0 , 0 6 6 0 3} * * * \\
0,02096 \\
\end{array}$ & $\begin{array}{c}-\mathbf{0 , 1 3 7 8 0} * * * \\
0,02777 \\
\end{array}$ \\
\hline Restricciones Institucionales & $\begin{array}{c}-\mathbf{0 , 0 8 0 3 2} * * \\
0,02307 \\
\end{array}$ & $\begin{array}{c}\mathbf{0 , 2 7 8 7 2} * * * \\
0,08768 \\
\end{array}$ \\
\hline Ln Magnitud de Distrito & $\begin{array}{c}\mathbf{0 , 0 8 0 3 2} * * \\
0,02307 \\
\end{array}$ & $\begin{array}{c}\mathbf{0 , 2 7 8 7 2} * * * \\
0,08768 \\
\end{array}$ \\
\hline Concentración Geográfica Desempleados (IHT) & $\begin{array}{c}\mathbf{0 , 2 2 5 0 8} * * * \\
0,04607\end{array}$ & \\
\hline Concentración Geográfica Desempleados (IHT)* Magnitud Distrito & $\begin{array}{c}-\mathbf{0 , 1 3 9 3 2} * * * \\
0,01805 \\
\end{array}$ & \\
\hline Concentración Geográfica Desempleados (EG) & & $\begin{array}{c}\mathbf{0 , 6 2 4 9 0} * * * \\
0,14244 \\
\end{array}$ \\
\hline Concentración Geográfica Desempleados $(E G) *$ Magnitud Distrito & & $\begin{array}{c}-\mathbf{0 , 0 9 5 6 5} * * * \\
0,02082\end{array}$ \\
\hline Observaciones & 187 & 204 \\
\hline R. Cuadrado & 0,97 & 0,98 \\
\hline
\end{tabular}

NOTA: Panel corrected standard errors debajo de los parámetros. Constante no mostrada, *** $\mathrm{p}<0,01, * * \mathrm{p}<0,05$, $* \mathrm{p}<0,1$. 


\section{CUADRO 2}

\section{ESTIMACIONES DE PENSIONES DE JUBILACIÓN}

\begin{tabular}{|c|c|c|}
\hline Variable Dependiente: Pensiones de Jubilación (\% PIB) & Modelo 2.1 & Modelo 2.2 \\
\hline Variable Dependiente Retardada & $\begin{array}{c}\mathbf{0 , 6 3 7 6 9} * * * \\
0,04758 \\
\end{array}$ & $\begin{array}{c}\mathbf{0 , 5 8 8 0 0} * * * \\
0,04816\end{array}$ \\
\hline Tasa Desempleo & $\begin{array}{c}-\mathbf{0 , 0 4 8 9 3} * \\
0,0292\end{array}$ & $\begin{array}{c}-\mathbf{0 , 0 4 3 5 2} \\
0,04348\end{array}$ \\
\hline Tasa Desempleo Cuadrática & $\begin{array}{c}\mathbf{0 , 0 0 3 1 0} * * \\
0,00126 \\
\end{array}$ & $\begin{array}{c}\mathbf{0 , 0 0 3 6 0} * \\
0,00201 \\
\end{array}$ \\
\hline Mayores 65 Años & $\begin{array}{c}\mathbf{0 , 2 3 2 4 9} \\
0,603\end{array}$ & $\begin{array}{c}-\mathbf{0 , 3 5 0 0 1} * * \\
0,19169\end{array}$ \\
\hline Mayores 65 Años Cuadrático & $\begin{array}{c}\mathbf{0 , 0 0 7 3 2} \\
0,522 \\
\end{array}$ & $\begin{array}{c}\mathbf{0 , 0 1 1 2 5} * * \\
0,00565\end{array}$ \\
\hline Tasa Interés & $\begin{array}{l}\mathbf{- 0 , 0 1 1 1} \\
0,02043\end{array}$ & $\begin{array}{c}-\mathbf{0 , 0 2 7 6 3} * * * \\
0,01404\end{array}$ \\
\hline Deuda Pública (año anterior) & $\begin{array}{c}\mathbf{0 , 0 0 3 7 1} * * \\
0,0018\end{array}$ & $\begin{array}{c}\mathbf{0 , 0 0 6 4 2} * * * \\
0,00155\end{array}$ \\
\hline Incremento PIB per capita & $\begin{array}{c}\mathbf{- 0 , 0 4 7 6 2} * * * \\
0,0131\end{array}$ & $\begin{array}{c}-\mathbf{0 , 0 5 4 2 3} * * * \\
0,01097\end{array}$ \\
\hline Ln PIB per capita & $\begin{array}{c}\mathbf{- 2 , 9 6 7 8 7} * * \\
1,11532\end{array}$ & $\begin{array}{c}-\mathbf{3 , 1 2 2 3 2} * * * \\
0,79907\end{array}$ \\
\hline Apertura Comercial & $\begin{array}{c}-\mathbf{0 , 0 0 3 9 3} * * \\
0,00234\end{array}$ & $\begin{array}{c}-\mathbf{0 , 0 0 1 8 8} \\
0,00259\end{array}$ \\
\hline Participación Electoral & $\begin{array}{c}\mathbf{0 , 0 0 4 8 8} * * * \\
0,00239\end{array}$ & $\begin{array}{c}-\mathbf{0 , 0 0 3 7 2} * * * \\
0,00208\end{array}$ \\
\hline Año Electoral & $\begin{array}{c}\mathbf{0 , 0 0 5 6 8} \\
0,0244 \\
\end{array}$ & $\begin{array}{c}-\mathbf{0 , 0 0 3 8 4} \\
0,02432\end{array}$ \\
\hline Gobierno Coalición & $\begin{array}{l}\mathbf{0 , 0 7 9 4 4} \\
0,09191 \\
\end{array}$ & $\begin{array}{l}\mathbf{0 , 0 8 9 8 7} \\
0,09829 \\
\end{array}$ \\
\hline Gobierno Minoría & $\begin{array}{l}\mathbf{0 , 0 6 1 3 1} \\
0,07435 \\
\end{array}$ & $\begin{array}{l}\mathbf{0 , 0 5 5 8 1} \\
0,08062 \\
\end{array}$ \\
\hline Gobierno de Izquierdas & $\begin{array}{c}-\mathbf{0 , 0 0 0 6 1} * \\
0,00055\end{array}$ & $\begin{array}{c}-\mathbf{0 , 0 0 0 3 2} \\
0,00043\end{array}$ \\
\hline Coordinación Salarial & $\begin{array}{c}\mathbf{- 0 , 0 4 2 3 5} \\
0,03614\end{array}$ & $\begin{array}{c}\mathbf{- 0 , 0 3 3 6 6} \\
0,02901\end{array}$ \\
\hline Restricciones Institucionales & $\begin{array}{c}-\mathbf{0 , 0 1 8 0 1} \\
0,03235 \\
\end{array}$ & $\begin{array}{c}-\mathbf{0 , 0 3 4 5 1} * * \\
0,02214\end{array}$ \\
\hline Ln Magnitud de Distrito & $0,36956 * * *$ & $0,19017 * * *$ \\
\hline Concentración Geográfica Mayores 65 (IHT) & $\begin{array}{c}\mathbf{0 , 3 0 6 4 6} * * * \\
0,0499 \\
\end{array}$ & \\
\hline Concentración Geográfica Mayores 65 (IHT)* Magnitud Distrito & $\begin{array}{c}-\mathbf{0 , 1 2 5 9 0} * * * \\
0,02998\end{array}$ & \\
\hline Concentración Geográfica Mayores 65 (EG) & & $\begin{array}{c}\mathbf{0 , 1 4 6 1 2} * * * \\
0,06797\end{array}$ \\
\hline Concentración Geográfica Mayores 65 (EG)*Magnitud Distrito & & $\begin{array}{c}-\mathbf{0 , 0 3 5 3 0} * * \\
0,01879\end{array}$ \\
\hline Observaciones & 187 & 204 \\
\hline R. Cuadrado & 0.98 & 0.98 \\
\hline
\end{tabular}

NOTA: Panel corrected standard errors debajo de los parámetros. Constante no mostrada, $* * * \mathrm{p}<0,01, * * \mathrm{p}<0,05$, $* \mathrm{p}<0,1$. 
En los cuadros que hemos presentado, los parámetros son condicionales. Para evaluar su importancia en todo el rango de valores de la variable independiente, seguimos las pautas de Brämbor et al. (2006) y suministramos los resultados en forma gráfica. Esto nos permite una visión más precisa sobre la magnitud del efecto.

El Gráfico 1 muestra el efecto del índice de Elison y Glaeser (EG) de concentración de desempleo condicionado a los distintos valores de la magnitud del distrito (modelo 1.2). El Gráfico 2 muestra la misma interacción para pensiones (modelo 2.2). Como se puede observar, el efecto marginal de la concentración del desempleo y los mayores de 65 difiere fuertemente a lo largo de todo el rango de proporcionalidad del sistema electoral. Una transición de un sistema mayoritario de distritos uninominales a uno perfectamente proporcional con un único distrito nacional (simulado aquí como los Países Bajos), implica un impacto diferencial de la concentración de desempleo de un 0,3 por 100 del PIB en prestaciones por desempleo. Teniendo en cuenta que en los países de la OCDE la media de las prestaciones por desempleo desde 1970 es de 1,3 por 100 del PIB, el efecto es muy relevante. El impacto es aún más importante en el caso de las pensiones. El efecto marginal de la concentración de personas mayores de 65 en un sistema mayoritario como el Reino Unido será casi un 0,8 por 100 del PIB mayor que en un sistema proporcional extremo, como los Países Bajos. Por lo tanto, los resultados de Persson y Tabellini quedan totalmente revertidos. Cuando los receptores están concentrados, los sistemas mayoritarios son más propensos a la formulación de políticas sociales.

\section{GRÁFICO 1}

EFECTO DE LA CONCENTRACIÓN DE MAYORES DE 65 SEGÚN PROPORCIONALIDAD DEL SISTEMA ELECTORAL

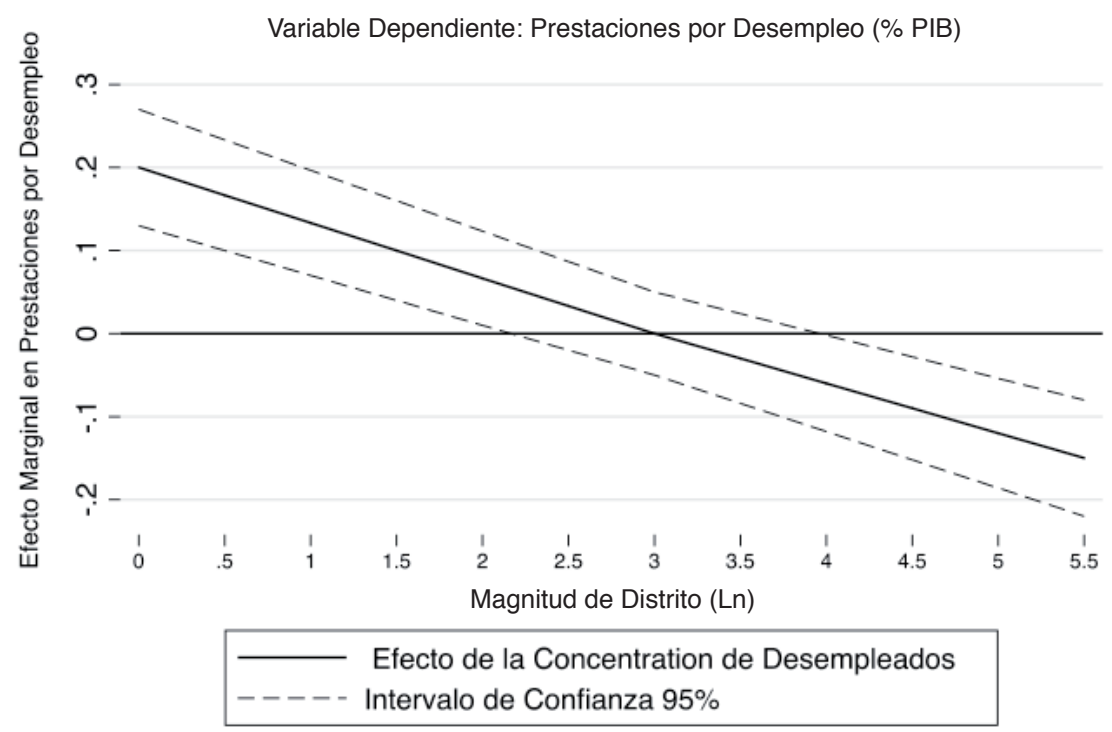




\section{GRÁFICO 2 \\ EFECTO DE LA CONCENTRACIÓN DE MAYORES DE 65 SEGÚN PROPORCIONALIDAD DEL SISTEMA ELECTORAL}

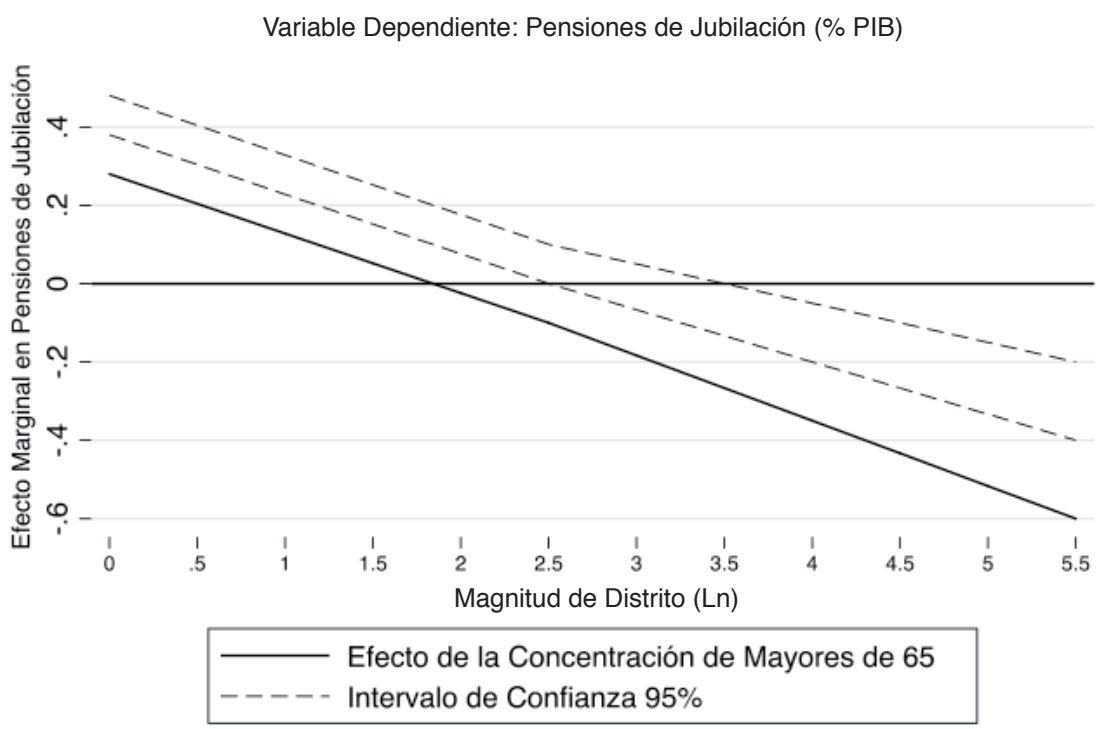

Teniendo en cuenta que en los países de la OCDE la media de las prestaciones por desempleo desde 1970 es de 1,3 por 100 del PIB, el efecto es muy relevante. El impacto es aún más importante en el caso de las pensiones. El efecto marginal de la concentración de personas mayores de 65 en un sistema mayoritario como el Reino Unido será casi un 0,8 por 100 del PIB mayor que en un sistema proporcional extremo, como los Países Bajos.

Ambos gráficos no solo muestran que en sistemas mayoritarios la concentración tiene un efecto más positivo que en proporcionales, sino que el efecto neto en estos últimos es negativo. Por lo tanto, los resultados de Persson y Tabellini quedan totalmente revertidos. Este efecto neto negativo en los sistemas proporcionales es un resultado novedoso y no contenido en el habitual marco teórico de los efectos de los sistemas electorales en las políticas de gasto social.

Por el contrario, cuando el sistema es proporcional, la provisión de la política social se reduce. En un sistema proporcional, en que todos los votos cuentan lo mismo independientemente de la ubicación de los votantes, la concentración debería ser irrelevante. Sin embargo, los modelos arrojan un efecto negativo y significativamente diferente de cero. Esto posiblemente implica que cuando un grupo social se concentra, se generan dos efectos. Por una parte, los ciudadanos de las regiones donde un grupo social se concentra desarrollan una fuerte preferencia por la política y su 
comportamiento electoral está fuertemente moldeado por los niveles de provisión. Por otro lado, en el resto de las regiones los ciudadanos desarrollan una oposición suave contra esa política, pues apenas vierte recursos en su entorno. Juntando ambos a nivel agregado, el efecto de la concentración podría ser ambiguo y se decantará de un lado u otro dependiendo de los incentivos institucionales. Así, sistemas electorales mayoritarios, donde ciertos distritos son más relevantes, incentivarían la provisión de la política social, ya que implica un pork barrel implícito a esos distritos. Sin embargo, podría ser que, cuando los partidos no están interesados en orientar las políticas a regiones concretas, los incentivos son, de hecho, a reducir la provisión de políticas, ya que la oposición suave es mayor que la demanda de los que están muy a favor.

\section{Conclusiones}

En este artículo se ha introducido una nueva variable pasada por alto en la literatura: la distribución geográfica de los beneficiarios de la políticas sociales. Los resultados muestran que esta variable es realmente importante para explicar la provisión de políticas en los países de la OCDE. Nuestros resultados muestran que existe un efecto positivo de la concentración de receptores en los sistemas electorales mayoritarios. Cuando un grupo social está relativamente más presente en determinadas regiones, los sistemas mayoritarios ofrecen mayores incentivos a la provisión en comparación con los proporcionales.

Estos resultados suponen una advertencia importante para la predicción de la literatura de sistemas electorales. La expectativa habitual de esta literatura es que la provisión de políticas sociales será menor en los sistemas electorales mayoritarios, porque los partidos tienen incentivos para concentrar sus esfuerzos distributivos en los distritos swing. Este artículo ha mostrado que este argumento está condicionado a la distribución de los beneficiarios de la política social. En los sistemas electorales mayoritarios, los partidos políticos están interesados en dirigir recursos a distritos específicos. Cuando los destinatarios de una política social se concentran en ciertos distritos, los partidos pueden de hecho canalizar más gasto a estos distritos mediante el gasto social.

En investigaciones futuras sería útil enriquecer estos resultados de varias maneras. En primer lugar, sería interesante analizar las implicaciones de estos resultados a nivel individual para comprobar hasta qué punto la región en la que vive un ciudadano influye en sus preferencias por políticas de gasto. Además, sería necesario poner a prueba si, aparte de las preferencias, el comportamiento electoral de los individuos responde a distintas motivaciones entre regiones. Los resultados han sugerido que en las regiones donde la política tiene un fuerte impacto, esta política será un asunto particularmente destacado en la evaluación electoral de los gobiernos. Por otro lado, habrá una oposición contra la política en regiones en las que esta implica una salida 
neta de recursos. Los análisis a nivel individual darían un fundamento teórico a estos resultados. Por último, a nivel macro, los resultados se enriquecerían con nuevos análisis con datos electorales a nivel de distrito. Sería interesante comprobar si la configuración social de los distritos swing tiene un efecto en el aumento de la provisión de políticas sociales.

\section{Referencias bibliográficas}

[1] ALESINA, ALBERTO y NOURIEL ROUBINI (1992): «Political Cycles in OECD Economies», The Review of Economic Studies 59:663-688.

[2] ALESINA, ALBERTO; NOURIEL ROUBINI, y GERALD D. COHEN (1998): Political cycles and the macroeconomy. Cambridge, Mass.: The MIT Press.

[3] ALTIG, DAVID y STEVE J. DAVIS (1989): «Government debt, redistributive fiscal policies, and the interaction between borrowing constraints and intergenerational altrusim», Journal of Monetary Economics, 24:3-29.

[4] ARMINGEON, KLAUS; SARAH ENGLER; MARLÈNE GERBER; PHILIPP LEIMGRUBER y MICHELLE BEYELER (2009): «Comparative Political Data Set 19602007», Institute of Political Science, University of Berne.

[5] BECK, NATHANIEL y JONATHAN N. KATZ (2004): «Time-Series-Cross-Section Issues: Dynamic». Manuscript.

[6] BOIX, CARLES (1998): Political parties, growth and equality: Conservative and social democratic economic strategies in the world economy. Cambridge University Press.

[7] BRADLEY, DAVID; EVELYNE HUBER; STEPHANIE MOLLER, FRANÇOIS NIELSEN y JOHN STEPHENS (2003): «Distribution and Redistribution in Postindustrial Democracies», World Politics, 55 (January): 193-228.

[8] BRAMBOR, THOMAS; WILLIAM ROBERTS CLARK y MATT GOLDER (2006): «Understanding Interaction Models: Improving Empirical Analyses», Political Analysis 14:63-82.

[9] CHANG, ERIC C. C.; MARK ANDREAS KAYSER y RONALD ROGOWSKI (2008): «Electoral Systems and Real Prices: Panel Evidence for the OECD Countries, 19702000», British Journal of Political Science, 38:739-751.

[10] COX, GARY (1990): «Centripetal and Centrifugal Incentives in Electoral Systems», American Journal of Political Science, 34:903-35.

[11] ELLISON, GLENN y EDWARD L. GLAESER (1997): «Geographic Concentration in U.S. Manufacturing Industries: A Dartboard Approach», Journal of Political Economy, 105:889-927.

[12] ESPING-ANDERSEN, GOSTA (1990): The Three Worlds of Welfare Capitalism. Princeton, NJ: Princeton University Press.

[13] EZCURRA, ROBERTO y MANUEL RAPUN (2006): «Regional Disparities and National Development Revisited: The Case of Western Europe», European Urban and Regional Studies 13:355-369.

[14] FRANZESE, ROBERT J. y IRFAN NOORUDDIN (2004): «The Effective Constituency in (Re)Distributive Politics: Alternative Bases of Democratic Representation, Geographic versus Partisan», Juan March Working Paper Series. 
[15] GOLDER, MATT (2007): Replication data for: Democratic Electoral Systems Around the World, 1946-2000.

[16] GRANT, WYN (1985): The Political economy of corporatism. London: Macmillan.

[17] GROSSMAN, GENE M. y ELHANAN HELPMAN (2006): «A Protectionist Bias in Majoritarian Politics», Quarterly Journal of Economics, 120:1239-1282.

[18] HALL, PETER y DAVID SOSKICE (2001): «Introduction», in Varieties of Capitalism: the Institutional Foundations of Comparative Advantage, Oxford: Oxford University Press.

[19] HIBBS, DOUGLAS A., Jr. (1977): «Political Parties and Macroeconomic Policy», The American Political Science Review, 71:1467-1487.

[20] HUBER, EVELYNE y JOHN D. STEPHENS (2001): Development and Crisis of the Welfare State: Parties and Policies in Global Markets. Chicago: University of Chicago Press.

[21] JONES, BRYAN D.; JAMES L. TRUE y FRANK R. BAUMGARTNER (1997): «Does Incrementalism Stem from Political Consensus or from Institutional Gridlock?», American Journal of Political Science 41:1319-1339.

[22] KATZENSTEIN, PETER J. (1985): Small states in world markets : industrial policy in Europe. Ithaca: Cornell University Press.

[23] KENWORTHY, LANE (2001): «Wage-setting measures», World Politics 54.1, 57-98.

[24] KENWORTHY, LANE y JONAS PONTUSSON (2005): «Rising Inequality and the Politics of Redistribution in Affluent Countries», Perspectives on Politics, 3:449-471.

[25] KORPI, WALTER (1978): The working class in welfare capitalism : work, unions, and politics in Sweden. London: Routledge \& Kegan Paul.

[26] KORPI, WALTER (1983): The democratic class struggle. London: Routledge \& Kegan Paul.

[27] LIJPHART, AREND (1994):. Electoral Systems and Party Systems: A Study of TwentySeven Democracies, 1945-1990, Comparative European Politics. Oxford: Oxford University Press.

[28] LIPSET, SEYMOUR M. y STEIN ROKKAN (1967): Party Systems and Voter Alignments: Cross-National Perspectives, New York: The Free Press.

[29] LIZZERI, ALESSANDRO y NICOLA PERSICO (2001): «The Provision of Public Goods under Alternative Electoral Incentives», The American Economic Review 91:225239.

[30] MILESI-FERRETTI, GIAN MARIA; ROBERTO PEROTTI y MASSIMO ROSTAGNO (2002): «Electoral Systems and Public Spending», Quarterly Journal of Economics, 117:609-657.

[31] NELSON, PHILLIP (1999): «Redistribution and the income of the median voter», $P u$ blic Choice 98:187-194.

[32] OCDE (2007): Social and Welfare Statistics.

[33] OVERMAN, HENRY G. y DIEGO PUGA (2002): «Unemployment Clusters across Europe's Regions and Countries», Economic Policy 17, no. 34: 115-48.

[34] PERSSON, TORSTEN y GUIDO ENRICO TABELLINI (2003): The economic effects of constitutions. Cambridge, Mass. ; London: MIT Press.

[35] PERSSON, TORSTEN; GUIDO ENRICO TABELLINI y ISABELLE BROCAS (2000): Political economics : explaining economic policy. Cambridge, Mass.; London: MIT Press. 
[36] PLUMPER, THOMAS y VERA E. TROEGER (2007): «Efficient Estimation of TimeInvariant and Rarely Changing Variables in Finite Sample Panel Analyses with Unit Fixed Effects», Political Analysis, 15:124-139.

[37] PONTUSSON, JONAS y DAVID RUEDA (2010): «The politics of inequality: Voter mobilization and left parties in advanced industrial states», Comparative Political Studies, 43.6: 675-705.

[38] RICKARD, STEPHANIE J. (2006): «Examining the Impact of Electoral Institutions on the Form of (Re)distributive Policies», Paper presented at the MPSA Conference 2006.

[39] RODRIK, DANI (1998): «Why Do More Open Economies Have Bigger Governments?», Journal of Political Economy, 106:997-1032.

[40] RUEDA, DAVID (2007): Social democracy inside out : partisanship and labor market policy in industrialized democracies. Oxford; New York: Oxford University Press.

[41] RUEDA, DAVID y JONAS PONTUSSON (2000): «Wage inequality and varieties of capitalism», World Politics, 52.3: 350-383.

[42] SCHMIDT, MANFRED G. (1996): «When parties matter: A review of the possibilities and limits of partisan influence on public policy», European Journal of Political Research, 30:155-183.

[43] SCHMITTER, PHILIPPE C. (1974): «Still the century of corporatism?», Rev. Polit. 36:85-131.

[44] SCRUGGS, LYLE (2005): Comparative Welfare Entitlements Dataset.

[45] TAAGEPERA, REIN y MATTHEW SOBERG SHUGART (1989): Seats and votes: The effects and determinants of electoral systems. New Haven: Yale University Press.

[46] TANZI, VITO (2002): «Globalization and the Future of Social Protection», Scottish Journal of Political Economy, 49, no. 1: 116-27.

[47] VISSER, JELLE (2009): ICTWSS: Database on Institutional Characteristics of Trade Unions, Wage Setting, State Intervention and Social Pacts in 34 countries between 1960 and 2007. 